\title{
Pulmonary artery pressure variation in patients with connective tissue disease: 24 hour ambulatory pulmonary artery pressure monitoring
}

\author{
D A Raeside, G Chalmers, J Clelland, R Madhok, A J Peacock
}

\begin{abstract}
Background-The specific contribution of secondary pulmonary hypertension to the morbidity and mortality of patients with underlying lung disease can be difficult to assess from single measurements of pulmonary artery pressure. We have studied patients with secondary pulmonary hypertension using an ambulatory system for measuring continuous pulmonary artery pressure (PAP). We chose to study patients with connective tissue disease because they represent a group at high risk of pulmonary vascular disease, but with little disturbance of lung function.

Methods-Six patients (five with progressive systemic sclerosis and one with systemic lupus erythematosis) were studied. They underwent preliminary cardiopulmonary investigations followed by Doppler echocardiography, right heart catheterisation, and ambulatory pulmonary artery pressure monitoring to measure changes in pressure over a 24 hour period including during a formal exercise test.
\end{abstract}

Results-All patients had pulmonary hypertension as measured by Doppler echocardiography with estimated pulmonary artery systolic pressures of 40$100 \mathrm{~mm} \mathrm{Hg}$. Pulmonary function testing revealed virtually normal spirometric values (mean $\mathrm{FEV}_{1} \mathbf{8 6 . 9 \%}$ predicted) but marked reduction in $\mathrm{CO}$ gas transfer factor (KCO $57.8 \%$ predicted). Exercise responses were impaired with mean $\mathrm{Vo}_{2} \max \quad 50.6 \%$ predicted. Ambulatory PAP monitoring indicated significant changes in pressures with variation in posture and activity throughout 24 hours. Resting PAP did not predict the change in PAP seen on exercise.

Conclusion-Conventional methods of assessment of the pulmonary circulation based on single measurements in the supine position may underestimate the stresses faced by the right side of the circulation. This ambulatory system allows monitoring of pulmonary haemodynamics continuously over 24 hours during normal activities of daily living. These measurements may increase our understanding of the contribution made by secondary pulmonary hypertension to the morbidity and mortality of the underlying lung disease.

(Thorax 1998;53:857-862)
Keywords: secondary pulmonary hypertension; connective tissue disease; Doppler echocardiography; cardiac catheterisation; micromanometer tipped pulmonary artery catheter; ambulatory pulmonary pressure monitoring; ambulatory pressure variations; pulse pressure

Pulmonary hypertension can complicate many diseases of the heart or lungs but there has been debate about its role in the mortality and morbidity of patients with primary lung disease. We have little understanding of the epidemiology of pulmonary hypertension in these conditions because, unlike the systemic circulation, there is no easily applied sphygmomanometer for the pulmonary circulation, with the consequence that the development of pulmonary hypertension can be missed in the early stages and its progression can be difficult to monitor. The currently available methods of measurement in the pulmonary circulation have some disadvantages. Echocardiography may be non-invasive, accurate and repeatable, but gives only an estimate of systolic pressure at rest or on limited exercise and can be technically difficult in some patients, especially those with chronic obstructive pulmonary disease (COPD). Cardiac catheterisation with fluid filled catheters has long been considered to be the gold standard but the position (lying supine on the catheter table) and the circumstances (at rest) during which the measurements are made may not reflect the situation in which an individual experiences symptoms.

In an attempt to overcome this difficulty, some authors have exercised patients while catheterised to reveal changes in pulmonary haemodynamics apparent only with increased cardiac output. However, even when measurements are made on exercise, the catheterisation procedure allows measurements to be made only over a short period of time and may not give information which represents accurately the pressures experienced during normal activities such as exercise and change in posture. Furthermore, cardiac catheters of the Swan Ganz type do not measure instantaneous pressures, the signal obtained may be damped by the fluid filled lumen, ${ }^{1}$ and it is difficult to maintain the necessary fixed relationship between the position of the external transducer and the right atrium.

We have attempted to address some of these difficulties by using (for the first time in this group of patients) a micromanometer tipped pulmonary artery catheter which can continuously record pulmonary artery pressure (PAP) in various postures and during different activi-
16 March 1998

Accepted for publication

26 May 1998 


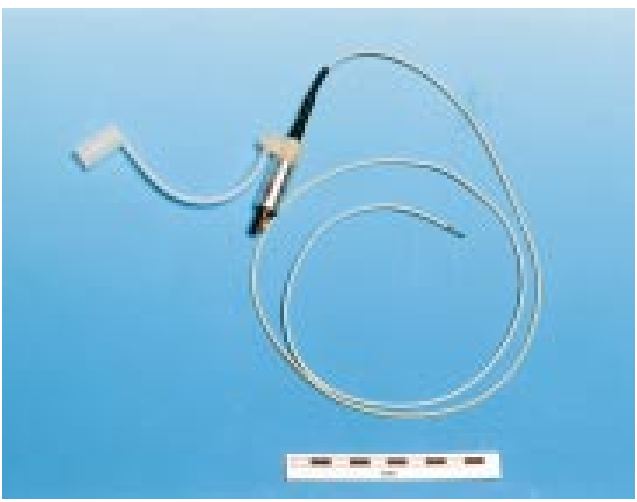

Figure 1 The micromanometer tipped pulmonary artery catheter. The external luer fitting allows injection of air for calibration. The stainless steel tip houses the transducer.

ties without loss of accuracy, and can make recordings for 24 hours. This allows collection of data of possibly greater relevance to the circumstances in which patients are likely to experience symptoms such as on exercise.

We wondered whether there are significant variations in pulmonary artery pressure with changes in posture and exercise in patients with pulmonary hypertension. To examine this hypothesis we studied the variations in pulmonary artery pressure over 24 hours, both at rest and on exercise, in a group of patients with connective tissue disease.

\section{Methods}

THE CATHETER

In this study we have used a $7 \mathrm{~F}$ catheter supplied by Gaeltech Ltd (Dunvegan, Isle of Skye, UK; fig 1). This catheter is designed for multiple use and is gas sterilised in ethylene oxide. It has a number of features which make it particularly useful for making ambulatory measurements in the pulmonary circulation including a micromanometer tip and an in vivo calibration system which allows correction for "zero drift". ${ }^{2}$ The catheter makes a continuous real time recording of the pulmonary artery pressure tracing and provides information about diastolic, mean, and systolic pulmonary artery pressures. There is no facility to measure cardiac output or pulmonary artery occlusion pressure at present, and the catheter is stiffer than a fluid filled catheter which may cause difficulties in placement, although we did not encounter this in our study. We did not experience any significant side effects from central venous cannulation or the placement of the catheters themselves, which remained in a satisfactory position throughout the 24 hour studies without requiring repositioning. This pressure monitoring system has previously been extensively described by Gibbs et $a l^{\beta}$ in patients with pulmonary hypertension secondary to heart disease. It has not been used before in a group of patients with pulmonary hypertension secondary to lung disease.

Prior to insertion the pressure transducer of the catheter was calibrated. The operating programme zeroed the pressure transducer before re-calibrating it against a measured pressure of $100 \mathrm{~mm} \mathrm{Hg}$. During recording the injection of $0.4 \mathrm{ml}$ of air via a syringe into the luer fitting on the catheter provided the necessary reference pressure to allow correction for zero drift. These corrections are required most frequently at the beginning of a recording when drift is most likely to occur. The calibration injections can be performed by the patient in the daytime and at night by the ward nursing staff, who are also able to confirm that the patient is asleep. Zero reference calibrations were carried out every hour throughout each study.

\section{DATA RECORDING}

The data are recorded by a portable battery powered recorder (Type MPR/2, Gaeltech Ltd, Isle of Skye, UK; fig 2) before being downloaded to a desk top computer for storage and analysis (Mitsubishi Apricot Pentium 100). The recorder incorporates a clock allowing annotation of the trace in real time. A button on the recorder, pushed by the patient, marks an event and time on the trace which can then be compared with the patient's activity diary (see below). This permits direct comparison of the pressure trace with activity. The memory (incorporating a $2 \mathrm{MB}$ expansion card) gives more than 24 hours continuous recording.

\section{PATIENT DIARY}

An important component of the recording system is the patient diary which consists of a simple paper record of time and current activity plus a note of when calibrations were made and the event button pressed. This diary can later be compared with the pulmonary artery pressure trace.

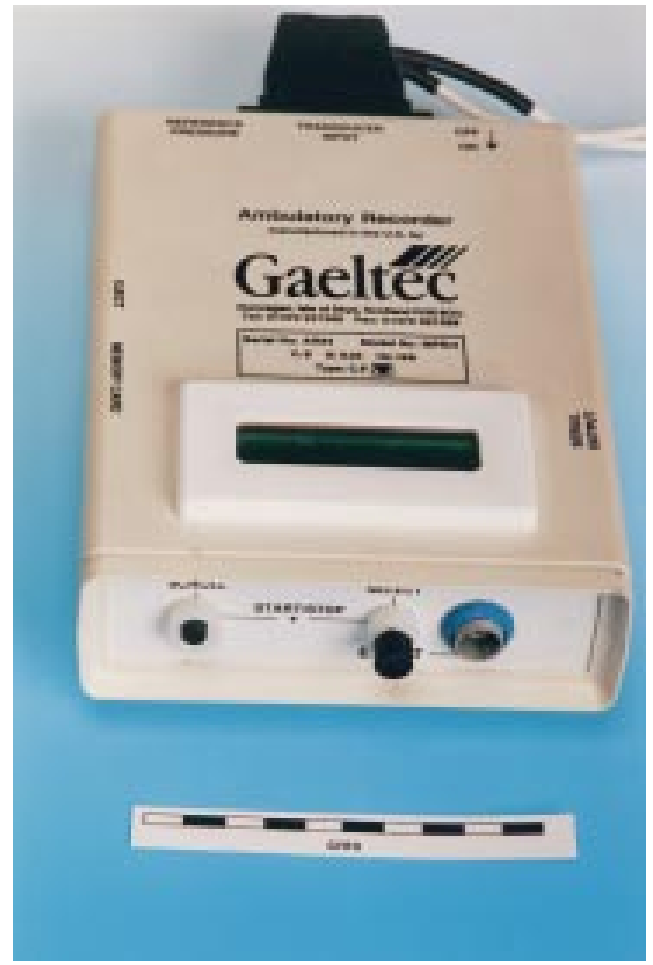

Figure 2 The ambulatory recorder. This weighs $650 \mathrm{~g}$ and is worn over the shoulder in a carrying case by the patient throughout the period of recording. During a recording the liquid crystal display on top of the box reads real time and percentage of memory occupied. The event button on the front of the recorder is pressed by the patient at the beginning and end of an activity or postural change, simultaneously with a calibration. 


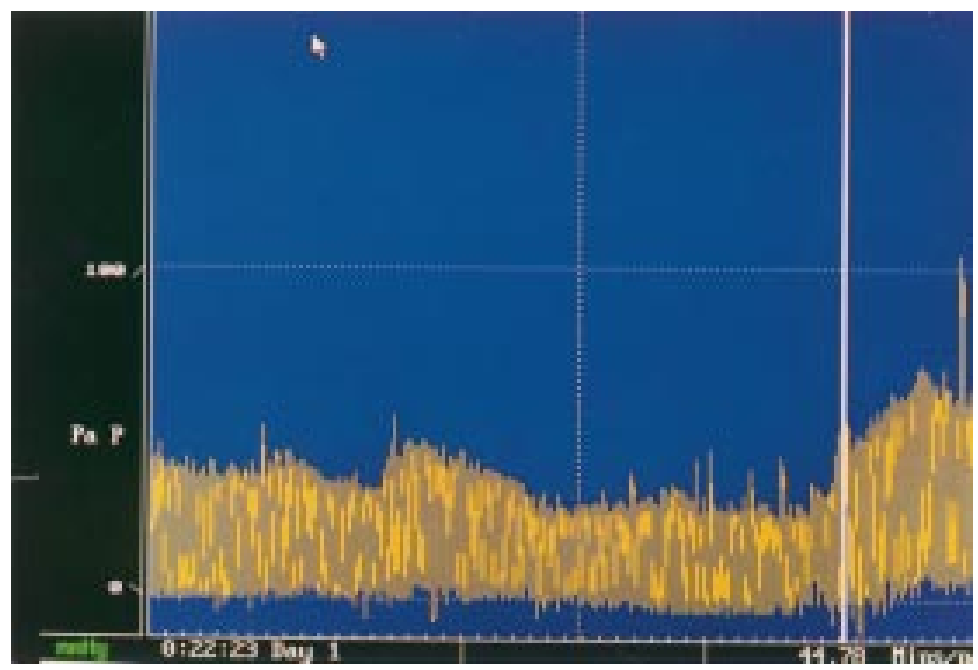

Figure 3 An ambulatory pressure trace of a patient with mild pulmonary hypertension recorded over 45 minutes. In the last 10 minutes of the recording the patient is undergoing an exercise test and there is a rise in pulmonary artery pressure (PAP).

DATA ANALYSIS

The analysis programme (Gaeltech, Isle of Skye, UK) incorporates software to permit correction of the entire pressure tracing for any drift relative to true zero. Segments of the recording can then be analysed as required using the timed trace of pulmonary artery pressure, and correlated with the times of a particular posture or activity recorded in the patient diary. The average systolic, diastolic, and pulse pressures for the entire trace and for each individual posture and activity can then be calculated by the analysis programme. Figure 3 shows a pressure trace. The standard deviations calculated from these measurements are small because the numbers of individual measurements are so large.

\section{STATISTICS}

Values are presented as mean (SD) unless otherwise stated. Differences between grouped mean pulmonary artery pressures measured in different postures were examined by analysis of variance (ANOVA) calculations, a $p$ value of $\leqslant 0.05$ being regarded as statistically significant.

\section{PATIENTS}

All six patients had connective tissue disease, as defined by immunopathological patterns, with antibody profiles suggestive of the categories stated in table 1, five with progressive systemic sclerosis (three with CREST variant (calcinosis, Raynaud's phenomenon, oesophageal dysmotility, sclerodactyly, telangectasia), and one with systemic lupus erythematosis). There were two

Table 1 Patient demographics

\begin{tabular}{llclllc}
\hline Patient no. & Age & Sex & Diagnosis & $\begin{array}{l}F E V_{1} \\
(\% \text { predicted })\end{array}$ & $\begin{array}{l}\text { KCO } \\
(\% \text { predicted })\end{array}$ & $\begin{array}{l}\text { Vo max } \\
\text { (\% predicted) }\end{array}$ \\
\hline 1 & 59 & F & PSS & 102.0 & 79.7 & 17 \\
2 & 66 & F & PSS & 101.6 & 45.1 & 45 \\
3 & 31 & F & SLE & 78.1 & 80.6 & 58 \\
4 & 68 & M & PSS & 94.4 & 53.9 & 45 \\
5 & 64 & F & PSS & 69.6 & 11.8 & 37 \\
6 & 62 & M & PSS & 75.6 & 42.7 & N/A \\
Mean (SD) & & & & $86.9(14.2)$ & $57.8(17.7)$ & $50.6(12.3)$ \\
\hline
\end{tabular}

$\mathrm{FEV}_{1}=$ forced expiratory volume in one second; $\mathrm{KCO}=$ carbon monoxide gas transfer coefficient; $\mathrm{Vo}_{2}$ max $=$ maximal oxygen uptake; PSS = progressive systemic sclerosis; SLE = systemic lupus erythematosis. men and four women of mean age 58 years (range 31-68). This study did not include a control group as the invasive nature of the investigation would have made this ethically difficult.

This investigation was approved by the local ethics committee and all patients gave written informed consent.

\section{MEASUREMENTS}

In each case pre-catheterisation investigations included a chest radiograph, electrocardiogram, pulmonary function tests, echocardiography, and a computed tomographic (CT) scan of the thorax to exclude other causes of secondary pulmonary hypertension. Parenchymal lung disease was excluded in our patients using high resolution CT scanning. Pulmonary artery systolic pressure was estimated from Doppler measurements. A symptom limited exercise test was also performed.

\section{Doppler echocardiography}

Echo Doppler measurements (Acuson 128 $\mathrm{XP} / 10 \mathrm{C}$, Acuson Ltd, Mount View, California, USA) were made in the semi-recumbent position. The jet of tricuspid regurgitation was identified, its maximum velocity measured, and pressure gradient calculated. ${ }^{4}$ This gradient was added to an assumed right atrial pressure $(10 \mathrm{~mm} \mathrm{Hg})$ to give an estimate of pulmonary artery systolic pressure. Echo measurements were made by the same operator in all cases 24 hours in advance of cardiac catheterisation, so the operator was ignorant of the "true" PAP. It was not possible logistically to make simultaneous measurements with echo Doppler and the micromanometer tipped pulmonary artery catheter.

\section{Cardiorespiratory exercise testing}

An exercise test (SensorMedics Corporation, Yorba Linda, California, USA) was carried out before cardiac catheterisation and repeated with the micromanometer tipped pulmonary artery catheter in situ. Initially patients performed a maximal exercise test on the cycle ergometer. During the repeat exercise test patients were exercised under steady state conditions at $30 \%$ of the maximum oxygen uptake $\left(\mathrm{VO}_{2} \mathrm{max}\right)$ achieved in the initial test. The exercise data presented are the mean pressures recorded at the onset of the fourth minute of steady state exercise.

\section{CARDIAC CATHETERISATION}

An introducer was placed in the right internal jugular vein and right heart catheterisation was performed using a triple channel thermodilution Swan Ganz catheter (Swan Ganz 7 F Thermodilution Catheter, Baxter Healthcare, Irvine, California, USA). The transducer was levelled with the right atrium with the patient lying supine. Pressure measurements for comparison with the other systems were made in the proximal pulmonary artery. Pressure values were recorded while patients breath held at functional residual capacity (to eliminate the effects of respiration on PAP). A mean of six beats was recorded and systolic and diastolic pressures were averaged. 
Table 2 Individual values of systolic pulmonary artery pressure (PAP) measured by three techniques (Doppler echocardiography, cardiac catheterisation with fluid filled Swan Ganz catheter, and micromanometer tipped pulmonary artery catheter while lying awake)

\begin{tabular}{|c|c|c|c|}
\hline Patient no. & $\begin{array}{l}\text { Echo systolic } \\
\text { PAP (mm Hg) }\end{array}$ & $\begin{array}{l}\text { Catheter } \\
\text { laboratory } \\
\text { systolic PAP } \\
\text { (mm Hg) }\end{array}$ & $\begin{array}{l}\text { Ambulatory } \\
\text { catheter systolic } \\
\text { PAP (mm Hg) }\end{array}$ \\
\hline 1 & 40 & 34 & 37 \\
\hline 2 & 70 & 65 & 59 \\
\hline 3 & 68 & 90 & 101 \\
\hline 4 & 88 & 90 & 117 \\
\hline 5 & 100 & 85 & 98 \\
\hline 6 & 70 & 90 & 99 \\
\hline Mean (SD) & 72.7 (20.4) & $75.8(22.6)$ & $85.2(30.4)$ \\
\hline
\end{tabular}

The lying awake value from the micromanometer tipped pulmonary artery catheter was chosen because that is the posture in which conventional cardiac catheterisation measurements are made.

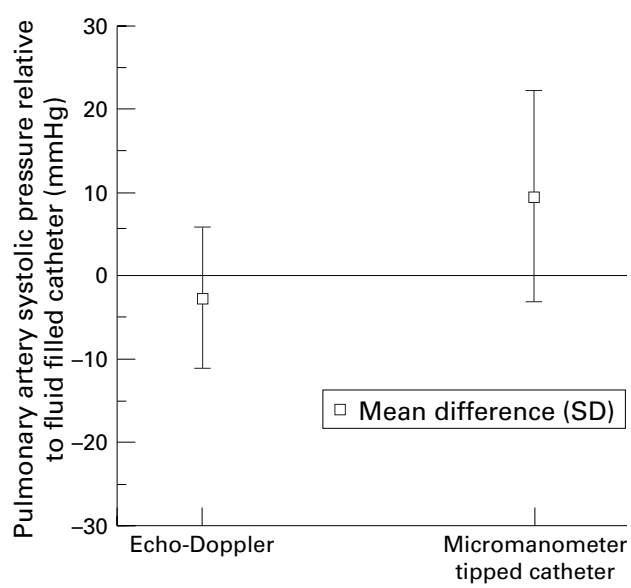

Figure 4 Differences in the values obtained for systolic PAP by Doppler echocardiography and micromanometer tipped pulmonary artery catheter from the values obtained by the fluid filled catheter. The differences between the three techniques are not significant.

AMBULATORY PULMONARY ARTERY PRESSURE MONITORING

Following cardiac catheterisation the Swan Ganz catheter was removed and the micromanometer tipped pulmonary artery catheter was calibrated as described above. It was placed in the pulmonary artery under fluoroscopic screening and left in situ for 24 hours. During this period the patient carried out normal daily activities in addition to a number of preset postural manoeuvres performed according to instructions from the investigator. These consisted of periods of 10 minutes each of lying

Table 3 Mean ambulatory pulmonary artery pressure (PAP) measured with the micromanometer tipped pulmonary artery catheter and heart rate averaged over 24 hours and during 10 minutes in each posture (lying asleep, lying awake, sitting and standing) and during exercise

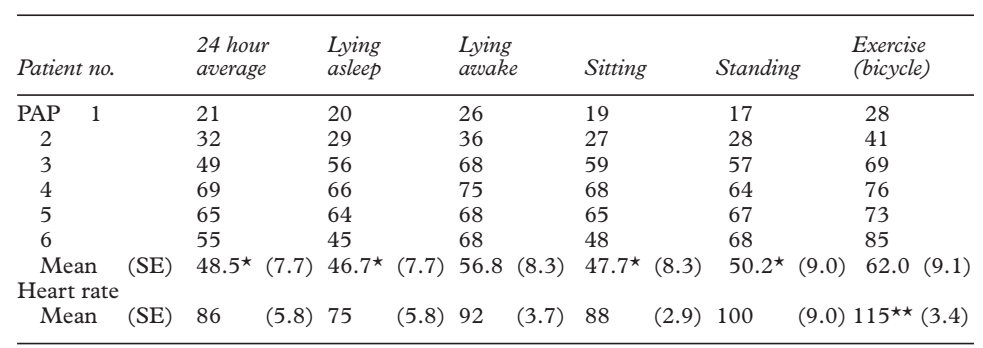

The exercise values were obtained at the beginning of the fourth minute of a steady state exercise test at $30 \%$ measured $\mathrm{VO}_{2} \max$.

${ }^{\star}$ PAP significantly different from lying awake $(p<0.05$, ANOVA)

$\star \star$ Heart rate significantly different from lying awake $(\mathrm{p}<0.05$, ANOVA). awake, sitting, and standing in that order, performed 2-4 hours after returning from the cardiac catheterisation laboratory. During the final hour a second exercise test was carried out with the catheter in situ, as described above.

At the end of the study the ambulatory recorder was stopped and the data it had collected downloaded to the hard disk of the personal computer. The pulmonary artery catheter was then removed under aseptic conditions on the general ward and the patient discharged home that evening. Following removal the micromanometer tipped pulmonary artery catheter was washed in antiseptic solution and its calibration rechecked. The catheter was connected via plastic tubing and the three way tap to a sphygmomanometer and a transducer calibration tube. When sealed, this allows a known pressure to be applied to the transducer tip via the sphygmomanometer using a syringe connected to the three way tap. The resultant real time pressure trace is recorded on screen and saved. The value measured and recorded in this way, if not equal to $100 \mathrm{~mm} \mathrm{Hg}$, indicated the error which had occurred during recording, and was then used to apply a correction factor to the entire pressure trace prior to analysis.

\section{Results}

BASELINE INVESTIGATIONS

These are shown in table 1. Pulmonary function tests revealed virtually normal spirometric values with a mean forced expiratory volume in one second $\left(\mathrm{FEV}_{1}\right)$ of $86.9 \%$ predicted but marked impairment in gas transfer (mean (SD) carbon monoxide transfer coefficient (KCO) $0.93(0.4) \mathrm{mmol} / \mathrm{min} / \mathrm{kPa} / \mathrm{l}$, $57.8 \%$ predicted (range 11.8-80.6)). All of the five patients who were able to complete a cardiopulmonary exercise test demonstrated impaired exercise responses (mean $\mathrm{VO}_{2} \max =$ $50.6 \%$ predicted, range $17-58)$.

\section{MEASUREMENT OF RESTING PAP BY THREE} METHODS

A comparison of values obtained using three methods of measurement of PAP is shown in table 2. Systolic PAP measured by echocardiography and micromanometer tipped pulmonary artery catheter is compared with systolic PAP obtained using the fluid filled catheter in fig 4. There was no significant difference between the values obtained for systolic PAP using these three methods.

\section{HEART RATES}

Heart rates were obtained from the 24 hour recordings and are shown in table 3. The expected increase with exercise and fall during sleeping was observed.

AMBULATORY PAP RECORDING

Ambulatory PAP was measured over 24 hours in all subjects and the results are shown in table 3 and fig 5. We observed that changes in PAP on exercise were not predicted by resting values of PAP. A higher PAP occurred while lying awake than when sitting or standing. There were significant variations in mean PAP for the group 


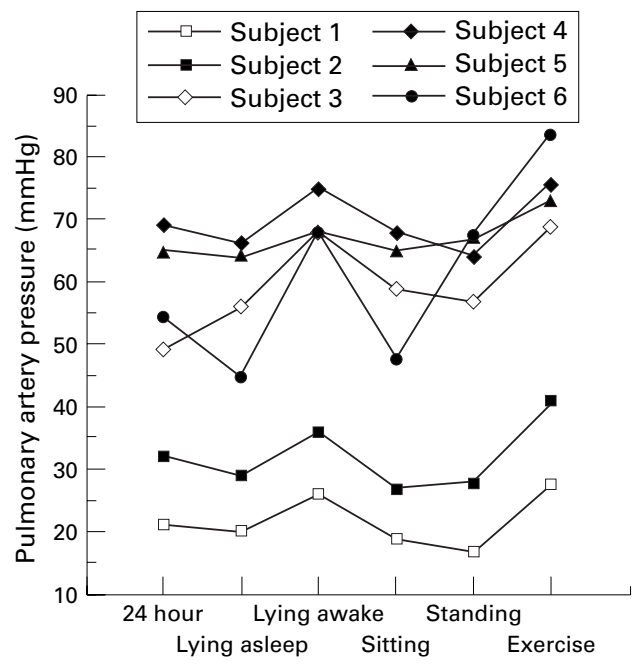

Figure 5 The mean pulmonary artery pressures recorded by micromanometer tipped pulmonary artery catheter for each subject for the total recording period of 24 hours and measured during 10 minutes in each posture (lying asleep, lying awake, sitting and standing). The exercise values were obtained at the beginning of the fourth minute of a steady state exercise test at $30 \%$ measured $\mathrm{VO}_{2}$ max.

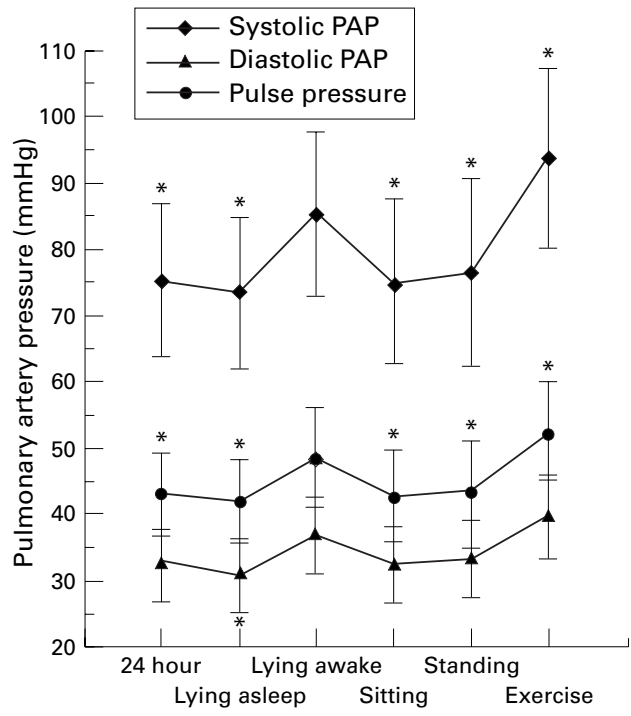

Figure 6 Mean systolic, diastolic, and pulse pulmonary artery pressures recorded by the micromanometer tipped pulmonary artery catheter for the group of subjects for each posture averaged over 24 hours and for each posture averaged over 10 minutes. The exercise values are the average of those obtained at the beginning of the fourth minute of a steady state exercise test at $30 \%$ measured $V_{O_{2}}$ max. The asterisks indicate that the values are significantly different from the measurement when lying awake.

between different postures compared with lying awake (fig 6). Lying awake was chosen for this comparison because it is the position in which conventional measurements are made.

\section{Discussion}

In this study we have shown that ambulatory 24 hour PAP monitoring in patients with connective tissue disease is feasible in the clinical setting. Our data indicate that PAP varied with different postures and activities and that mean PAP in these patients fell on sitting up or standing compared with lying awake.

While it may not be surprising that a physiological variable such as pulmonary artery pressure changes over 24 hours, these variations have not been measured previously in these patients. We have chosen "lying awake" as the baseline measurement for comparison with the other postures because it is the position in which conventional pressure measurements are usually made. We observed that the change in PAP seen on exercise was not predicted by the resting PAP (measured lying awake) in this group of patients with varying levels of pulmonary hypertension. The patient group in this study had reduced exercise tolerance compared with that predicted (table 1). A number of explanations have been proposed for this.

Previous work in patients with connective tissue disease using Doppler echocardiography has shown reduced exercise capacity, thought to be due in part to a rise in pulmonary vascular resistance on exercise, ${ }^{5}$ while other authors have highlighted the importance of pulmonary artery haemodynamics during exercise and the consequent increase in right ventricular workload in patients with primary pulmonary hypertension. ${ }^{6}$ Our data suggest that, not only are there significant variations in pressure with exercise, even in patients with only mild resting pulmonary hypertension, but there are also pressure variations during normal daily activity. This may indicate that the stresses faced by the right ventricle over a 24 hour period may also vary considerably and that conventional methods of measurement of PAP may underestimate this variation. A number of issues arise from these observations: (1) PAP while lying awake is higher than when measured sleeping in the same position; (2) PAP falls on taking up a sitting or standing posture; (3) PAP variation appears to be due to changes mainly in systolic, and hence pulse, pressure; (4) PAP at rest does not predict the change in PAP on exercise.

\section{PAP lying awake}

The higher values of PAP when lying awake compared with lying asleep suggest that the former may not truly reflect the basal state. Values when lying awake were always measured after at least 10 minutes at rest and this position was the first of the series of postures in which measurements were made after return to the main ward from the cardiac catheterisation laboratory. During the lying awake manoeuvre patients were observed continuously and were discouraged from moving or speaking. At night they were asked to lie flat with the identical number of pillows as had been in place when lying awake, and were checked hourly by the nursing staff (when performing in vivo calibration of the catheter) who noted whether the patients were asleep or not. Despite these precautions, in the absence of continuous EEG monitoring it is not possible to say that these patients were asleep throughout the night.

\section{Change in PAP with posture}

Micromanometer tipped catheter values also show a fall in PAP on sitting or standing. Doppler echo values (obtained in the semirecumbent position) were also lower than the fluid filled catheter baseline values, but we did not repeat the Doppler measurements in the lying position. This observation has been 
reported previously by Brecker et al who found that, in patients with severe pulmonary hypertension, Doppler echocardiography consistently underestimated systolic PAP by $20 \%$ when compared with high fidelity pulmonary artery catheters. Though Doppler values were lower in our study, they were not significantly so, possibly because our patient group had milder pulmonary hypertension.

These differences may be explained by the fact that these two techniques are measuring different things; however, the lower values measured with Doppler echocardiography may be due to decreased venous return on sitting or standing, with a subsequent fall in right ventricular filling pressure and hence cardiac output.

\section{Change in PAP with exercise}

$\mathrm{PAP}$ at rest did not predict changes in PAP with exercise in this group of patients. This is unlike the situation in patients with moderate or severe pulmonary hypertension in whom such a relationship is described. ${ }^{8}$ This anomaly may be due to the fact that the exercise workload at which pressures were measured differed between our subjects.

Patients with similar lung function often have markedly dissimilar exercise tolerance and we speculate that these differences may be because of variation in the response of the pulmonary circulation to increased cardiac output. If the resting pulmonary artery pressures in patients with mild pulmonary hypertension are not predictive of exercise induced changes in PAP, then the earlier diagnosis of the development of changes in the pulmonary vasculature may be possible using the micromanometer tipped catheter than is the case with conventional methods of measurement.

In the 1980s there was a move away from single "office" readings of systemic blood pressure to the development of simple noninvasive systems for the measurement of 24 hour ambulatory systemic blood pressure. ${ }^{9}$ Clearly the technique described in this paper is not likely to gain widespread acceptance in the investigation of the pulmonary circulation because of its cost and invasiveness. Individual catheters cost approximately $£ 800$ each (though are reusable) and the recording box about $£ 10000$, but as a research tool it does offer a similar advantage to ambulatory blood pressure monitoring in the systemic circulation in that it can provide data about pressure in relation to daily activity and symptoms. The mean PAP over 24 hours can be accurately calculated and some additional insight gained into the chronic haemodynamic load faced by the right ventricle. This may allow the earlier identification of individuals at greater risk of subsequent right ventricular failure and consequently those with a poorer prognosis.

The advantages of ambulatory PAP measurement may also be relevant in the assessment of response to treatment. At present there is no completely selective pulmonary vasodilator and there continues to be debate about the appropriateness of treatment in secondary pulmonary hypertension. There is, however, considerable evidence to support the use of vasodilators in primary pulmonary hypertension. ${ }^{10}$ Objective assessment of responses to these treatments will be required and, in addition to the information which may be gained by conventional catheter laboratory measurements of the pulmonary circulation, the micromanometer tipped pulmonary artery catheter described in this paper can add measurements of 24 hour variations in PAP and the changes seen on exercise, and so possibly present a fuller picture of the continuing stresses faced by the right ventricle as well as responses to treatment. Furthermore, it is likely that treatment may be more effective early in the development of pulmonary vascular changes when single resting measurements may be normal ${ }^{11}$ and the PAP response to exercise may reveal that these changes are taking place.

This study demonstrates some of the strengths and weaknesses of this technique in the assessment of the pulmonary circulation in patients with connective tissue disease. Although ambulatory monitoring of PAP has been extensively used in patients with heart failure $^{12}$ and coronary artery disease, ${ }^{13}$ it has not been used previously in patients with respiratory disease and larger numbers of patients are required to assess its future role. However, we believe that it may have additional advantages alongside conventional systems in the assessment of the pulmonary circulation.

This study was supported by a British Heart Foundation project grant.

1 Naeije R. Pulmonary vascular function. In: Peacock AJ, ed. Pulmonary circulation, a handbook for clinicians. London: Chapman and Hall, 1996: 13-27.

2 Gibbs JSR, Cunningham D, Sparrow J, et al. Unpredictable zero drift in intravascular micromanometer catheters implications for catheter design. Cardiovasc Res 1989;23: implica.

3 Gibbs SR, MacLachlan D, Fox KM. A new system for ambulatory pulmonary artery pressure recording. Br Heart f 1992;68:230-5.

4 Yock PG, Popp RL. Non-invasive evaluation of right ventricular systolic pressure by Doppler ultrasound in patients with tricuspid regurgitation. Circulation 1984;70: 657-62.

5 Winslow TM, Ossipov M, Redberg RF, et al. Exercise capacity and haemodynamics in systemic lupus erythematosis: a Doppler echocardiographic exercise study. Am Heart F 1993;126:410-4

6 Laskey WK, Ferrari VA, Palevsky HI, et al. Pulmonary artery haemodynamics in primary pulmonary hyper-

7 Brecker SJD, Gibbs JSR, Fox KM, et al. Comparison of Doppler derived haemodynamic variables and simultaneous high fidelity pressure measurements in severe pulmonary hypertension. Br Heart $\mathcal{F}$ 1994;72:384-9.

8 Reeves J. Approach to the patient with pulmonary hypertension. In: Weir EK, Reeves JT, eds. Pulmonary hypertension. Mount Kisco, New York: Futura Publishing, 1984: 34 .

9 Brunner HR, Waeber B, Nussberger J. Clinical use of nonnvasive ambulatory blood pressure recording. F Hypertens 1985;3(Suppl 2):S13-17.

10 Rubin LJ. ACCP Consensus Statement. Primary pulmonary hypertension. Chest 1993;104:236-50.

11 Salvaterra CG, Brundage BH, Rubin LJ. Is the early diagnosis of pulmonary hypertension possible, useful, and cost sis of pulmonary hypertension possible, useful, and cost
effective? In: Weir EK, Archer SL, Reeves JT, eds. The diagnosis and treatment of pulmonary hypertension. Mount Kisco, nosis and treatment of pulmonary hypertension

12 Gibbs JSR, Cunningham D, Shapiro LM, et al. Diurnal variation of pulmonary artery pressure in chronic heart failure. $\mathrm{Br}$ Heart $\mathcal{F}$ 1989;62:30-5.

13 Levy RD, Shapiro LM, Wright C, et al. Haemodynamic response to myocardial ischaemia during unrestricted activity, exercise testing, and atrial pacing assessed by ambulatory pulmonary artery pressure monitoring. $\mathrm{Br}$ Heart f 1986;56:12-18. 